\title{
Designing Core-Shell 3D Photonic Crystal Lattices for Negative Refraction
}

\author{
Victoria F. Chernow*a, Ryan C. Ng ${ }^{\mathrm{b}}$, Julia R. Greer ${ }^{\mathrm{a}}$ \\ ${ }^{a}$ Division of Engineering and Applied Sciences, California Institute of Technology, Pasadena, CA, \\ 91125; ${ }^{\mathrm{b}}$ Division of Chemistry and Chemical Engineering, California Institute of Technology, \\ Pasadena, CA, 91125, USA;
}

\begin{abstract}
We use a plane wave expansion method to define parameters for the fabrication of 3-dimensional (3D) core-shell photonic crystals $(\mathrm{PhCs}$ ) with lattice geometries that are capable of all-angle negative refraction (AANR) in the midinfrared centered around $8.0 \mu \mathrm{m}$. We discuss the dependence of the AANR frequency range on the volume fraction of solid within the lattice and on the ratio of the low index core material to the high index shell material. Following the constraints set by simulations, we fabricate two types of nanolattice PhCs: (1) polymer core-germanium shell and (2) amorphous carbon core-germanium shell to enable experimental observation of $3 \mathrm{D}$ negative refraction and related dispersion phenomena at infrared and eventually optical frequencies.
\end{abstract}

Keywords: 3-dimensional photonic crystal, nanolattice, negative refraction, two-photon lithography, collimation

\section{INTRODUCTION}

Negative refraction of electromagnetic waves was first proposed by Veselago in $1968^{1}$. He theorized that if a material were to have simultaneously negative effective permittivity and permeability (a so called "left-handed" material), the energy flow in the material, as dictated by the Poynting vector, would be anti-parallel to the wave vector ${ }^{1}$. The implication of this property is that group velocity, $v_{g}=d \omega / d k$, would be negative, and a refracted wave propagating away from the interface of this material would travel on the negative side of the surface normal. Per Snell's law, a negative angle of refraction gives rise to a negative index of refraction ${ }^{2}$. More recently it was shown that negative refraction is also possible in dielectric photonic crystals $(\mathrm{PhCs})$ that are composed of purely right-handed materials ${ }^{3}$. The underlying physical principles for this phenomenon are based on complex Bragg scattering from the photonic lattice structure, in contrast to effective medium theory which treats left-handed materials as uniform media to determine their properties ${ }^{4}$.

In the case of photonic crystals, theory and experiments have largely focused on achieving negative refraction in twodimensional periodic structures, which is necessary for the fabrication of superlenses, which are capable of subwavelength imaging ${ }^{5,6}$. True subwavelength imaging of three-dimensional (3D) objects however, requires a 3D PhC capable of negatively refracting light traveling in all three spatial planes, and it was not until 2002 that Luo, Johnson, and Joannopoulos (ref. 7) proposed a lattice capable of negative refraction for all incident angles from air, now known as allangle negative refraction (AANR) ${ }^{7}$.

The structure proposed by Luo et al. consists of a body-centered cubic (bcc) lattice of air cubes embedded in a dielectric with $\varepsilon=18$ (e.g. germanium in the near-infrared region) ${ }^{7}$. It is specified that for a conventional bcc unit cell with periodicity $a$, the sides of the air cube are parallel to the unit cell, and have edge length of $0.75 a^{7}$. This PhC lattice yields a band structure that possesses a negative photonic-mass region (mathematically, a concave-downward shape) in the third band between the $\Gamma H$ and $\Gamma N$ directions, where AANR is observed and the effective refractive index of the PhC is $\mathrm{n}_{\mathrm{eff}} \approx-1$ (refer to Figure 1 in reference 7 ). The frequency range for AANR in this lattice spans from $0.375(2 \pi c / a)$ to $0.407(2 \pi c / a)$, where the equi-frequency contours of the third band are all-convex (circular) and larger than that of air (Figure 2 in reference 7). The lower boundary of this AANR region falls at the frequency where the band has an inflection point, and the upper boundary is at the frequency where the band intersects the light line (dispersion curve for air) along the $\Gamma H$ direction.

*vchernow@caltech.edu; phone 1626 395-4416;

Photonic and Phononic Properties of Engineered Nanostructures VII, edited by Ali Adibi,

Shawn-Yu Lin, Axel Scherer, Proc. of SPIE Vol. 10112, 101120G · @ 2017 SPIE

CCC code: $0277-786 \mathrm{X} / 17 / \$ 18 \cdot$ doi: $10.1117 / 12.2251545$ 
Luo et al. proposed and theoretically investigated this 3D PhC capable of AANR, yet, the only experimental demonstration of AANR in 3D has been shown by Lu et al., at microwave frequencies ${ }^{8,9}$. The lack of experiments demonstrating 3D negative refraction in the optical and infrared range is due, in large part, to the difficulties inherent in fabricating fully 3D lattices with features on the nano- and micrometer length scale. With the advent and refinement of two-photon lithography (TPL) direct laser writing (DLW), it is now possible to fabricate arbitrarily complex 3D structures with sub-micron features. TPL DLW allows for the printing of low index polymeric scaffolds, and this process, coupled with chemical vapor deposition (CVD) or sputtering, for the deposition of high index dielectric materials like germanium $(\mathrm{Ge})$, provides a prospective route for the fabrication of 3D core-shell lattices capable of negative refraction. This process has not yet been explored for the creation of AANR lattices; in this paper we present numerical calculations to identify the effect of parameters like beam diameter, which directly translates into volume fraction $f$, and the ratio of low index core (polymer or amorphous carbon) to high index (Ge) shell material, have on the resulting frequency range available for negative refraction.

\section{SIMULATION PARAMETERS AND DISCUSSION}

We use a plane wave expansion method (PWEM) to calculate photonic band-structure and equi-frequency contours (EFCs) of 3D PhCs capable of AANR in the mid-infrared $(7.6 \mu \mathrm{m}-8.6 \mu \mathrm{m})$ wavelength range using a bcc lattice analogous to the one proposed by Luo $\mathrm{et}^{\mathrm{al}} \mathrm{l}^{7}$. These simulations were performed using the commercial software package RSOFT BandSOLVE.
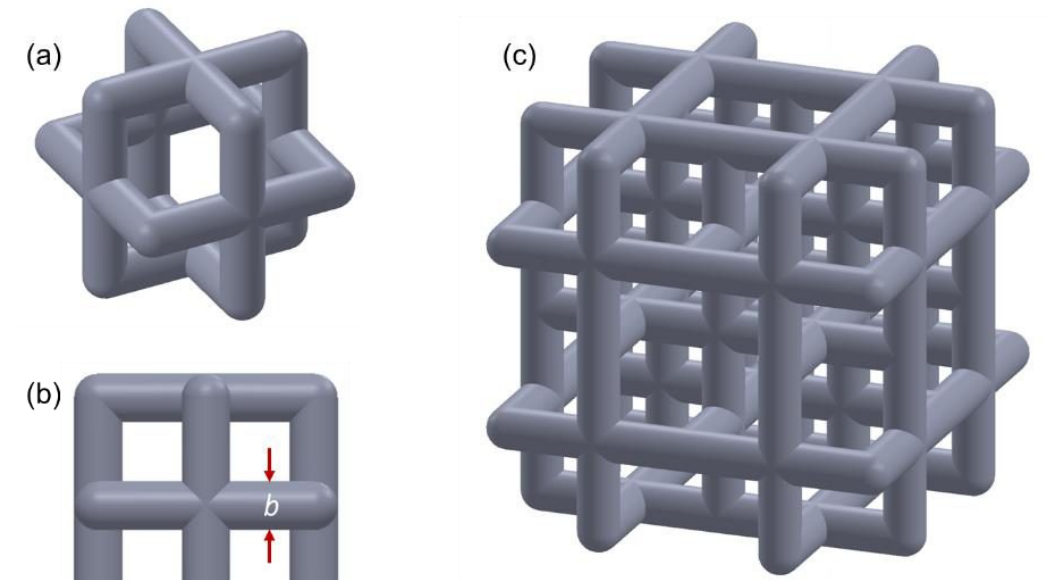

(b)

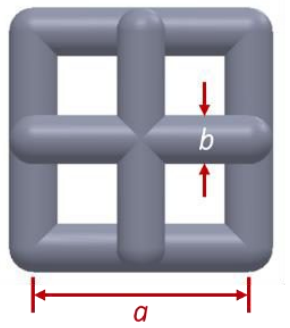

Figure 1. CAD schematic of the 3D bcc PhC lattice. (a) A view the PhC unit cell with cylindrical beams. (b) A single unit cell with representative dimensions for period, $a$, and beam diameter, $b$. (c) Schematic of the full, 3D lattice PhC.

Figure 1 shows such a representative lattice with cylindrical, rather than rectangular beams as used in Luo $e t$ al. ${ }^{7}$, to better reflect the topology of structures fabricated using TPL DLW.

In the first case study we investigate the effect of beam diameter, or volume fraction $f$, on the average frequency and frequency range for observing AANR. In this analysis, lattice beams are comprised of a single uniform material with a dielectric constant $\varepsilon=16.038$ (e.g., Ge in the mid-infrared around $\lambda=8.0 \mu \mathrm{m})^{10}$. The period of the bcc lattice is set to $a$ $=4 \mu \mathrm{m}$, an easily fabricated unit cell size using TPL, and the beam diameter is varied between $b=0.125 a=500 \mathrm{~nm}$, and $b=0.5 a=2 \mu \mathrm{m}$. This is equivalent to varying the volume fraction of the lattice between $f=0.07$ and 0.68 . 

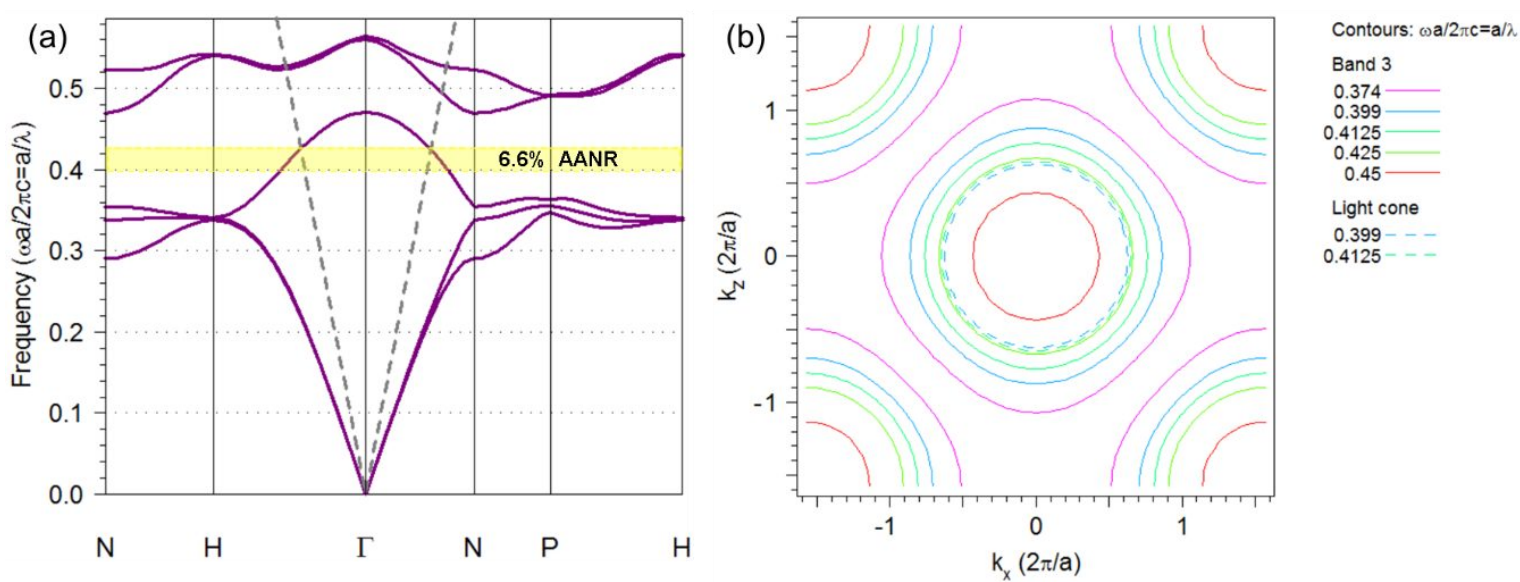

Figure 2. (a) Representative band structure of the 3D PhC $(f=0.23)$. The yellow shaded area represents the frequency range where the $\mathrm{PhC}$ exhibits negative refraction for incoming radiation of all angles. The dashed grey lines are the light lines along $\Gamma H$ and $\Gamma N$. (b) Slice of the equi-frequency surfaces on the $k_{y}=0$ plane for band 3 shown in part $(a)$. The highest frequency circle is colored red and lies at the center. Subsequently lower frequencies have larger diameter contours. The dashed circles are equi-frequency contours for air, and are lower than their PhC contour counterparts at the same frequency.

By applying the plane wave expansion method to Maxwell's equations, we solved for eigenfrequencies at a given wave vector and obtained results qualitatively similar to those of Luo et $\mathrm{al}^{7}$. Figure 2(a) shows a representative band structure of a $\mathrm{PhC}$ with a beam diameter $b=0.25 a(f=0.23)$. The dashed straight grey lines in Figure 2(a) are the light lines, or dispersion curves for air, along directions of high symmetry in the first Brillouin zone of a bcc crystal. Within the PhC, light propagates normal to its dispersion surface, which is the 3D k-space representation of the supported eigenmodes (bands). Taking a cross-section of a dispersion surface yields an equi-frequency contour (EFC) that can be used to predict the direction of light propagation in the PhC. Figure 2(b) shows such a cross-section of the dispersion surface for band 3 along the $k_{y}=0$ plane, generating EFCs. We observe that in the frequency range between $\omega=0.399(2 \pi c / a)$ to $0.426(2 \pi c / a)$ EFCs are circular and larger than the corresponding EFCs of air. As the frequency increases, EFC radii shrink, indicating negative group velocity, and consequently negative effective refractive index in this range.
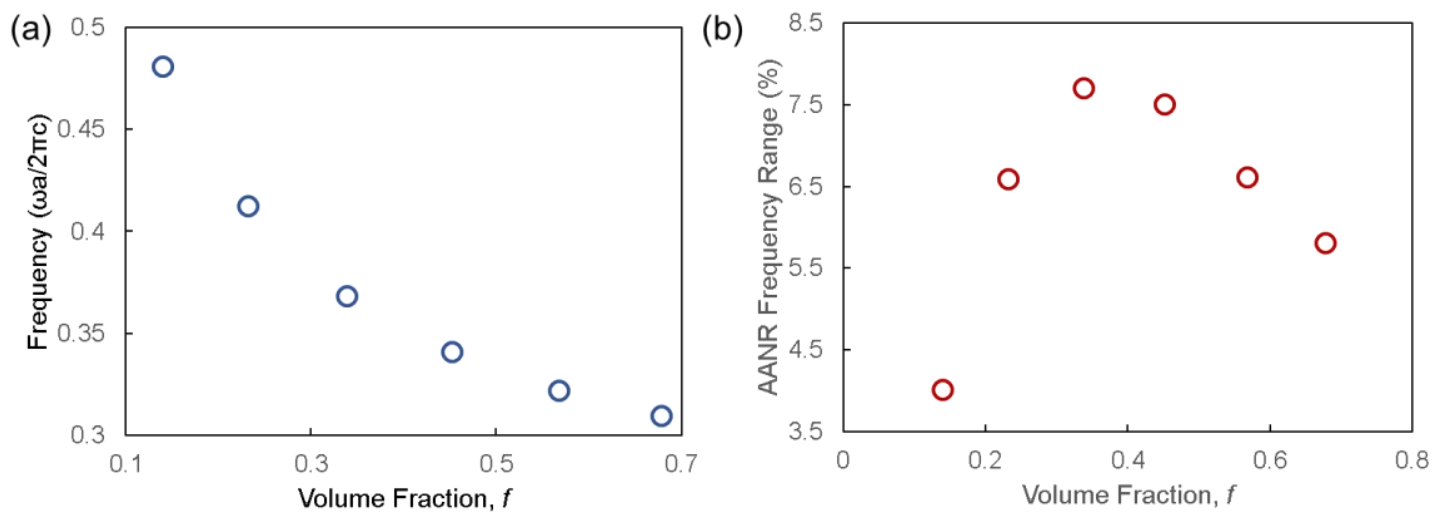

Figure 3. (a) Average frequency at which AANR is observed as a function of the volume fraction (beam diameter). (b) AANR frequency range, represented as a percentage difference, as a function of volume fraction.

Varying the volume fraction $(f)$ by changing the beam diameter for a lattice with a set periodicity, we observe that the average frequency for AANR decreases with increasing volume fraction (Figure 3(a)). This is the result of bands compressing and shifting to lower frequencies when the volume fraction of high index material in a PhC increases ${ }^{11}$. Figure 3(b) shows the relative AANR frequency range as a function of volume fraction. Unlike the average AANR frequency, which decreases monotonically with increasing $f$, (Figure 3(a)), the AANR frequency range has a more complex dependence on the volume fraction, reaching a maximum of $7.7 \%$ for a beam diameter $b=1.25 \mu \mathrm{m}(f=0.34)$. When the volume of high index material is too low, as in the case of $b=0.5 \mu \mathrm{m}(f=0.07)$, no negative refraction is 
observed. These calculations reveal that there exists both a minimum volume fraction of high index material necessary to begin discerning AANR, as well as an optimal volume fraction for maximizing the AANR frequency range. The aim of this paper is to identify physically realizable lattice parameters for the fabrication of PhCs with appreciable AANR in the wavelength range between $7.6 \mu \mathrm{m}-8.6 \mu \mathrm{m}$; we choose to focus on lattices with $b=1.0 \mu \mathrm{m}$, or $f=0.23$. For this volume fraction, the AANR frequency range is substantial, at $6.6 \%$, and for a lattice period of $4.0 \mu \mathrm{m}$, the average wavelength for observing AANR is centered around $9.7 \mu \mathrm{m}$ (Table 1). Though this wavelength lies outside the measurement capabilities of our mid-IR spectroscopic setup (which utilizes a quantum cascade laser operating between $7.6 \mu \mathrm{m}-8.6$ $\mu \mathrm{m})$, actual PhC fabrication efforts will involve using TPL DLW and sputter deposition to create polymer core, germanium shell lattices. The combined refractive index of the core-shell structure will be lower than that of pure Ge, and will push the AANR region to shorter wavelengths.

Table 1. Figures of merit derived from band structure and EFC calculations on PhC lattices of varied beam diameters with nbeam $=4.0047$.

\begin{tabular}{|l|l|l|l|l|l|}
\hline $\begin{array}{c}\text { Beam } \\
\text { Diameter } \\
(\mu \mathrm{m})\end{array}$ & $\begin{array}{c}\text { Volume } \\
\text { Fraction, } \\
f\end{array}$ & $\begin{array}{c}\text { Absolute } \\
\text { Frequency Range, } \\
\Delta \omega(2 \pi \mathrm{c} / \mathrm{a})\end{array}$ & $\begin{array}{c}\text { Average AANR } \\
\text { Frequency } \\
(2 \pi \mathrm{c} / \mathrm{a})\end{array}$ & $\begin{array}{c}\text { AANR } \\
\text { Frequency } \\
\text { Range }(\%)\end{array}$ & $\begin{array}{c}\text { Average AANR } \\
\text { Wavelength } \\
(\mu \mathrm{m})\end{array}$ \\
\hline 0.75 & 0.1394 & 0.0193 & 0.4808 & 4.0146 & 8.3203 \\
\hline 1.0 & 0.2320 & 0.0272 & 0.4124 & 6.5955 & 9.6993 \\
\hline 1.25 & 0.3381 & 0.0284 & 0.3682 & 7.7132 & 10.8637 \\
\hline 1.5 & 0.4517 & 0.0256 & 0.3409 & 7.5095 & 11.7336 \\
\hline 1.75 & 0.5670 & 0.0213 & 0.3221 & 6.6139 & 12.4204 \\
\hline 2.0 & 0.6781 & 0.0180 & 0.3098 & 5.8102 & 12.9116 \\
\hline
\end{tabular}

We also used PWEM to calculate band structure and EFCs to quantify the effect of modified dielectric constant on AANR frequency when core-shell structures are explored. For this analysis, lattice periodicity remains at $a=4 \mu \mathrm{m}$, the total beam diameter is set to $b=0.25 a=1 \mu \mathrm{m}(f=0.23)$, and the relative ratio of low-index $(\mathrm{n}=1.49)$ acrylate polymer core material ${ }^{12}$, and high-index $(n=4.0047) \mathrm{Ge}$ shell material ${ }^{10}$ is varied by progressively increasing the core diameter and simultaneously shrinking the thickness of the shell to maintain the same total beam diameter. We varied the core diameter from $b_{\text {core }}=0.1 b=100 \mathrm{~nm}$, where shell thickness $t_{\text {shell }}=\left(b-b_{\text {core }}\right) / 2=450 \mathrm{~nm}$, to $b_{\text {core }}=0.9 b=900 \mathrm{~nm}$, with $t_{\text {shell }}=\left(b-b_{\text {core }}\right) / 2=50 \mathrm{~nm}$, and in so doing decreased the compound index of the lattice (see Table 2$)$. We observed a monotonic increase in the average frequency for AANR and a monotonic decrease in AANR frequency range with decreasing compound refractive index of lattice beams (Figure 4(a-b)). As this ratio increases, the combined index of the whole $\mathrm{PhC}$ decreases down to a threshold index below which negative refraction is no longer possible. Specifically, Figure 4(b) shows that below an index of $\mathrm{n} \sim 2.49$, equivalent to a polymer core of $b_{\text {core }}=600 \mathrm{~nm}$ and germanium shell of $t_{\text {shell }}=200 \mathrm{~nm}$ in lattices of volume fraction $f=0.23$, an AANR region does not exist. It appears that below this index the periodic modulation effect is weak, and the EFC changes its shape from circular to square-like. Figure 4(c) shows the band 3 EFCs for a lattice with $b_{\text {core }}=700 \mathrm{~nm}$ and $t_{\text {shell }}=150 \mathrm{~nm}$, where $\mathrm{n}_{\text {beam }}=2.24$, and reveals that in the frequency region below the light line (which falls at $\omega=0.546(2 \pi c / a)$ for this lattice) the contours have a square-like shape. A circular EFC means that light can travel isotropically, and a square-like EFC, with its broad, flat regions, indicates that light can propagate without spreading or diffracting significantly, known as self-collimation. We found that lattices with a compound index $<2.49$ do not display negative refraction, but their EFCs indicate that such lattices possess the unique property of self-collimation. 

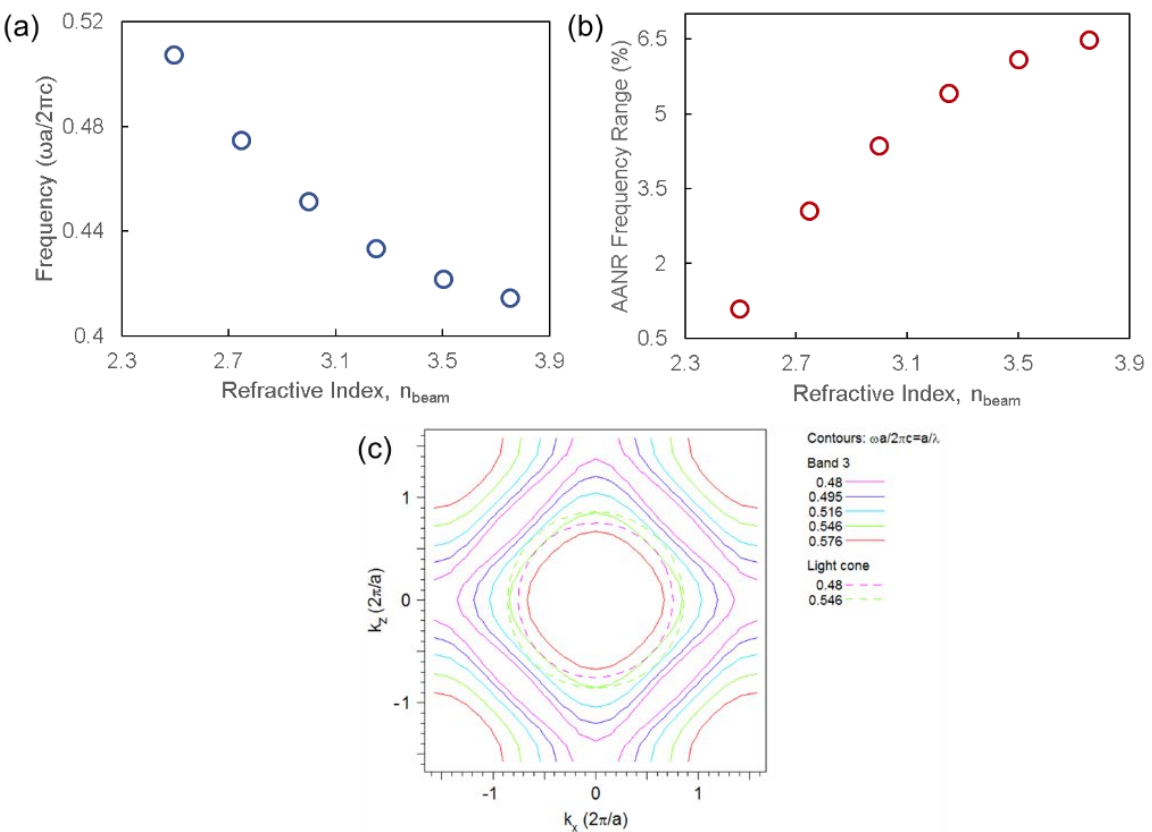

Figure 4. (a) Average frequency at which AANR is observed in the $\mathrm{PhC}$ when the compound refractive index of the lattice is varied (polymer core to Ge shell ratio changes). (b) AANR frequency range, represented as a percentage difference, for $\mathrm{PhCs}$ with varying compound refractive index of the lattice varied (polymer core to Ge shell ratio changes). (c) Slice of the equi-frequency surfaces on the $k_{y}=0$ plane for a PhC lattice with $b_{\text {core }}=700 \mathrm{~nm}$ and $t_{\text {shell }}=150 \mathrm{~nm}\left(\mathrm{n}_{\text {beam }}=2.24\right)$.

Table 2. Figures of merit derived from band structure and EFC calculations on core-shell PhC lattices of varied polymer core diameter and Ge shell thickness.

\begin{tabular}{|l|l|l|l|l|l|l|}
\hline $\begin{array}{c}\text { Core beam } \\
\text { diameter, } \\
\mathrm{b}_{\text {core }}(\mu \mathrm{m})\end{array}$ & $\begin{array}{c}\text { Shell } \\
\text { thickness, } \\
\mathrm{t}_{\text {shell }}(\mu \mathrm{m})\end{array}$ & $\begin{array}{c}\text { Refractive } \\
\text { Index, } \\
\mathrm{n}_{\text {beam }}\end{array}$ & $\begin{array}{c}\text { Absolute } \\
\text { Frequency } \\
\text { Range, } \Delta \omega \\
(2 \pi \mathrm{c} / \mathrm{a})\end{array}$ & $\begin{array}{c}\text { Average } \\
\text { AANR } \\
\text { Frequency } \\
(2 \pi \mathrm{c} / \mathrm{a})\end{array}$ & $\begin{array}{c}\text { AANR } \\
\text { Frequency } \\
\text { Range }(\%)\end{array}$ & $\begin{array}{c}\text { Average } \\
\text { AANR } \\
\text { Wavelength } \\
(\mu \mathrm{m})\end{array}$ \\
\hline 0.1 & 0.45 & 3.7532 & 0.0269 & 0.4145 & 6.4905 & 9.6513 \\
\hline 0.2 & 0.40 & 3.5018 & 0.0257 & 0.4217 & 6.0951 & 9.4865 \\
\hline 0.3 & 0.35 & 3.2503 & 0.0235 & 0.4336 & 5.4204 & 9.2262 \\
\hline 0.4 & 0.30 & 2.9988 & 0.0197 & 0.4515 & 4.3637 & 8.8603 \\
\hline 0.5 & 0.25 & 2.7474 & 0.0145 & 0.4749 & 3.0536 & 8.4237 \\
\hline 0.6 & 0.20 & 2.4959 & 0.0056 & 0.5076 & 1.1032 & 7.8802 \\
\hline
\end{tabular}

This series of simulations revealed that to preserve the circular shape of EFCs and to observe AANR between $7.6 \mu \mathrm{m}-$ $8.6 \mu \mathrm{m}$ using polymer-Ge core-shell lattices, the core diameter should be no greater than $\sim 600 \mathrm{~nm}$. This beam diameter is at a size scale below the resolution limit of TPL DLW capability (cylindrical beams are limited to a diameter of $\sim 900$ $\mathrm{nm})$.

As an alternative to writing lattices with a polymeric core, recent literature suggests that if a polymer lattice is pyrolyzed under specific temperature and atmospheric conditions, amorphous carbon (a-C) lattices with significantly smaller dimensions will be formed ${ }^{13}$. Amorphous carbon is lossy in the mid-infrared (the extinction coefficient $\kappa=1.8818$ at $\lambda=$ $8.0 \mu \mathrm{m}$ ) but has a higher refractive index of $\mathrm{n}=2.9622$ at $\lambda=8.0 \mu \mathrm{m}$ compared to the $\mathrm{n}=1.49$ of the acrylate polymer $^{14,12}$. To quantify the effect on average AANR frequency and frequency range when core-shell lattices are composed of a-C cores and Ge shells, we repeated the previous series of band structure and EFC simulations, replacing 
only the core polymer material parameters with those of a-C (Table 3). We find that as is the case in the polymer coreshell lattices, increasing the ratio of a-C to Ge material in the lattice decreases the overall combined index of the structure. We observe a monotonic increase in the average frequency for AANR and a monotonic decrease in AANR frequency range with decreasing compound refractive index of lattice beams (Figure 5(a-b)). Even at the extreme of $b_{\text {core }}$ $=900 \mathrm{~nm}$ and $t_{\text {shell }}=50 \mathrm{~nm}$, a small but apparent AANR frequency range exists since this lattice configuration has $n_{\text {beam }}$ $>2.49$. This suggests that fabricating a-C core, Ge shell lattices with core diameters between $700 \mathrm{~nm}$ and $900 \mathrm{~nm}$ would enable AANR observation in the ideal 7.6-8.6 $\mu \mathrm{m}$ range.
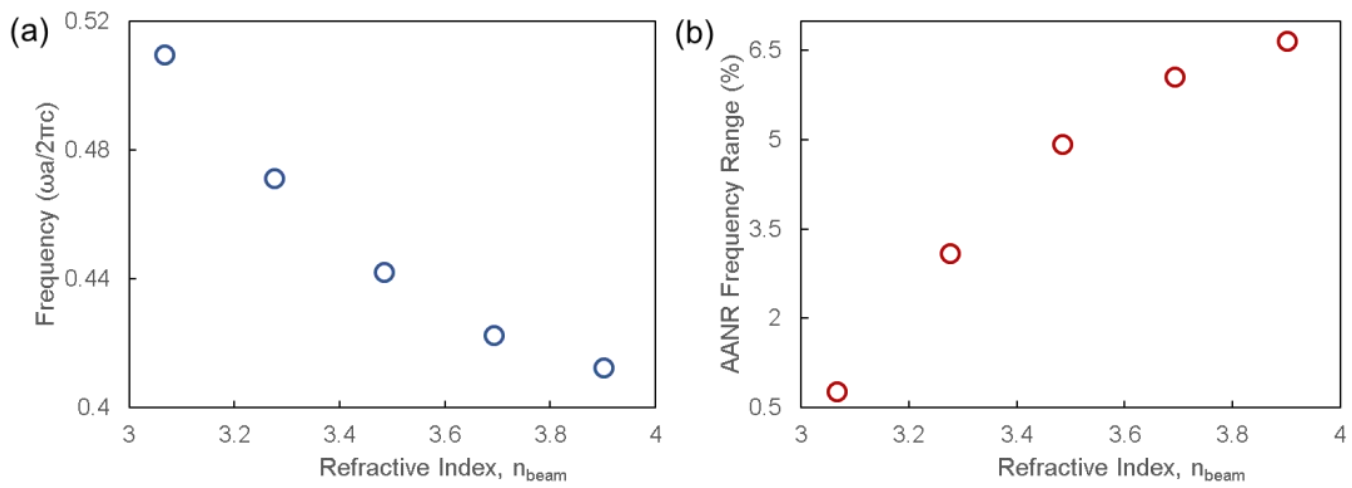

Figure 5. (a) Average frequency at which AANR is observed in the PhC when the compound refractive index of the lattice is varied (amorphous carbon core to Ge shell ratio changes). (b) AANR frequency range, represented as a percentage difference, for PhCs with varying compound refractive index of the lattice varied (amorphous carbon core to Ge shell ratio changes).

Table 3. Figures of merit derived from band structure and EFC calculations on core-shell PhC lattices of varied amorphous carbon core diameter and Ge shell thickness.

\begin{tabular}{|l|l|l|l|l|l|l|}
\hline $\begin{array}{c}\text { Core beam } \\
\text { diameter, } \\
\mathrm{b}_{\text {core }}(\mu \mathrm{m})\end{array}$ & $\begin{array}{c}\text { Shell } \\
\text { thickness, } \\
\mathrm{t}_{\text {shell }}(\mu \mathrm{m})\end{array}$ & $\begin{array}{c}\text { Refractive } \\
\text { Index, } \\
\mathrm{n}_{\text {beam }}\end{array}$ & $\begin{array}{c}\text { Absolute } \\
\text { Frequency } \\
\text { Range, } \Delta \omega \\
(2 \pi \mathrm{c} / \mathrm{a})\end{array}$ & $\begin{array}{c}\text { Average } \\
\text { AANR } \\
\text { Frequency } \\
(2 \pi \mathrm{c} / \mathrm{a})\end{array}$ & $\begin{array}{c}\text { AANR } \\
\text { Frequency } \\
\text { Range }(\%)\end{array}$ & $\begin{array}{c}\text { Average } \\
\text { AANR } \\
\text { Wavelength } \\
(\mu \mathrm{m})\end{array}$ \\
\hline 0.1 & 0.45 & 3.9005 & 0.0275 & 0.4125 & 6.6675 & 9.6981 \\
\hline 0.3 & 0.35 & 3.6920 & 0.0256 & 0.4226 & 6.0577 & 9.4652 \\
\hline 0.5 & 0.25 & 3.4835 & 0.0218 & 0.4420 & 4.9321 & 9.0498 \\
\hline 0.7 & 0.15 & 3.2750 & 0.0146 & 0.4712 & 3.0985 & 8.4890 \\
\hline 0.9 & 0.05 & 3.0665 & 0.0039 & 0.5095 & 0.7655 & 7.8516 \\
\hline
\end{tabular}

\section{SAMPLE FABRICATION AND MEASUREMENT}

Fabrication of core-shell AANR capable 3D PhCs is currently underway. TPL DLW has been used to fabricate polymer $\mathrm{PhC}$ nanolattices with a period of $4.0 \mu \mathrm{m}$ and beam diameter of $\sim 900 \mathrm{~nm}$ (Figure 6(a)). Isotropic etching methods including $\mathrm{O}_{2}$ plasma are being explored as a means of reducing beam diameter to $\sim 600 \mathrm{~nm}$. Figure 6(b) shows a focused ion beam (FIB) cross-section through a Ge coated polymer PhC nanolattice. Deposition of high index Ge has been accomplished using sputtering, and conformality of Ge thickness throughout the structure is being evaluated. AANR with core-shell PhCs will be measured using a custom built angle-resolved mid-IR spectrometer. This setup utilizes a quantum cascade laser operating between $7.6 \mu \mathrm{m}-8.6 \mu \mathrm{m}$ and allows for full control over sample and detector positioning via independent rotation stages. 

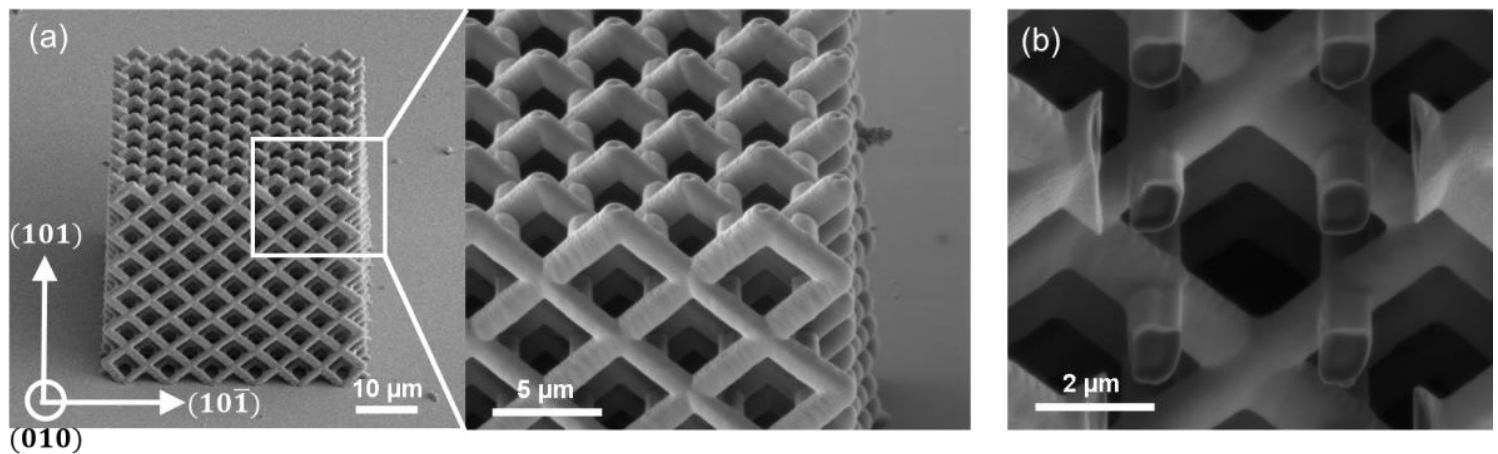

Figure 6. (a) Scanning electron microscopy (SEM) images of an as fabricated polymer 3D bcc PhC. It should be noted that lattices are fabricated such that the (101) surface of the $\mathrm{PhC}$ is exposed, for enhanced coupling efficiency with incoming light. ${ }^{7}$ (b) Focused ion beam (FIB) cross section through the (010) face of the core-shell PhC. FIB is used to evaluate the conformality of sputtered Ge on polymer beams throughout the lattice.

\section{SUMMARY}

We have performed PWEM calculations to investigate the effect of volume fraction and dielectric constant on the AANR average frequency and AANR frequency range in 3D bcc lattice photonic crystals. By performing a series of parameter sweeps we have identified that a volume fraction of $f=0.23$, which translates to a circular beam diameter of $1.0 \mu \mathrm{m}$ for a lattice with $4.0 \mu \mathrm{m}$ periodicity, is appropriate for the fabrication of low index core, high index shell lattice structures with AANR in the mid-infrared (7.6-8.6 $\mu \mathrm{m}$ range). These parameters provide useful guidelines for fabricating nanolattices with broad applications ranging from superlenses to functional integrated photonic circuit components. This work also demonstrates that the ratio of core-shell material is critical to modifying the very dispersion phenomena observed in the 3D photonic crystal. Tuning this core-shell ratio allows for a switch between negative refraction and selfcollimation properties, showcasing the versatility of 3D lattices in optical applications.

\section{REFERENCES}

[1] Veselago, V. G., "The electrodynamics of substances with simultaneously negative values of $\varepsilon$ and $\mu$," Sov. Phys. Uspekhi 10(4), 509-514 (1968).

[2] Luo, C., Johnson, S. G., Soljačić, M., Joannopouls, J. D.., Pendry, J. B., “Novel optical phenomena with photonic crystals," Proc. SPIE - Int. Soc. Opt. Eng. 5166, 207-219 (2004).

[3] Notomi, M., "Theory of light propagation in strongly modulated photonic crystals: Reflectionlike behavior in the vicinity of the photonic band gap," Phys. Rev. B 62(16), 10696 (2000).

[4] Baba, T., Matsumoto, T.., Asatsuma, T., "Negative Refraction in Photonic Crystals," Adv. Sci. Technol. 55, 91100 (2008).

[5] Luo, C., Johnson, S., Joannopoulos, J.., Pendry, J., “All-angle negative refraction without negative effective index," Phys. Rev. B 65(20), 2-5 (2002).

[6] Cubukcu, E., Aydin, K., Ozbay, E., Foteinopoulou, S.., Soukoulis, C. M., "Subwavelength resolution in a twodimensional photonic-crystal-based superlens.," Phys. Rev. Lett. 91(20), 207401 (2003).

[7] Luo, C., Johnson, S. G.., Joannopoulos, J. D., "All-angle negative refraction in a three-dimensionally periodic photonic crystal,” Appl. Phys. Lett. 81(13), 2352-2354 (2002).

[8] Lu, Z., Shi, S., Schuetz, C., Murakowski, J.., Prather, D., "Three-dimensional photonic crystal flat lens by full 3D negative refraction.," Opt. Express 13(15), 5592-5599 (2005).

[9] Lu, Z., Murakowski, J. A., Schuetz, C. A., Shi, S., Schneider, G. J.., Prather, D. W., "Three-dimensional subwavelength imaging by a photonic-crystal flat lens using negative refraction at microwave frequencies," Phys. Rev. Lett. 95(15), 2-5 (2005).

[10] Icenogle, H. W., Platt, B. C.., Wolfe, W. L., "Refractive indexes and temperature coefficients of germanium and silicon," Appl. Opt. 15(10), 2348-2351 (1976).

[11] Joannopoulos, J. D., Johnson, S. G., Winn, J. N.., Meade, R. D., Photonic crystals: molding the flow of light, Princeton University Press (2008). 
[12] Chernow, V. F., Alaeian, H., Dionne, J. A.., Greer, J. R., "Polymer lattices as mechanically tunable 3dimensional photonic crystals operating in the infrared," Appl. Phys. Lett. 107(10) (2015).

[13] Bauer, J., Schroer, A., Schwaiger, R.., Kraft, O., "Approaching theoretical strength in glassy carbon nanolattices.," Nat. Mater. 15(4), 438-443 (2016).

[14] Hagemann, H.-J., Gudat, W.., Kunz, C., "Optical constants from the far infrared to the x-ray region: $\mathrm{Mg}, \mathrm{Al}, \mathrm{Cu}$, Ag, Au, Bi, C, and Al2O3,” J. Opt. Soc. Am. 65(6), 742 (1975). 